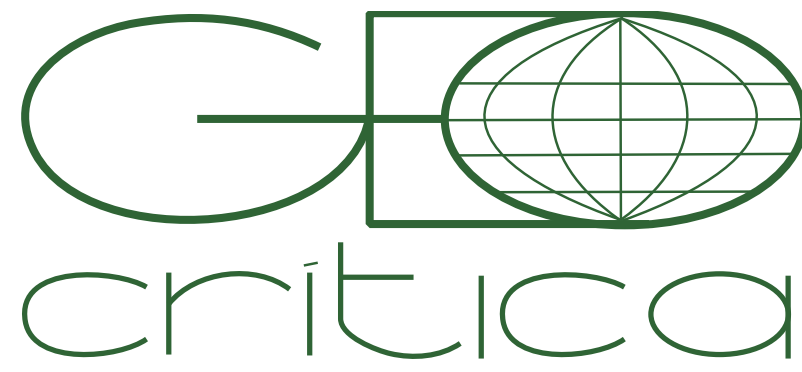

\section{Scripta Nova}

Revista Electrónica de Geografía y Ciencias Sociales Universitat de Barcelona

ISSN: 1138-9788

Vol. 25, Núm. 2 (2021), 37-56

\title{
LAS ALCALDESAS DE ROBADORS. RESISTENCIA, COMPROMISO Y VOZ DE LAS TRABAJADORAS SEXUALES DEL RAVAL DE BARCELONA
}

Este artículo se ha realizado a partir de un estudio de caso llevado a cabo en el marco del proyecto "En las fronteras de lo urbano: desposesión, resiliencia y resistencia en espacios urbanos extremos (RESDERES)" financiado por el Ministerio de Economía y Competitividad. (Proyectos I+D+ Convocatoria 2015: Programa Estatal de Investigación, Desarrollo e Innovación Orientada a los Retos de la Sociedad, Plan Estatal de Investigación Científica y Técnica y de Innovación 20162020). Ref. CSO2015-65066-R

\author{
Anna Clua \\ Universitat Oberta de Catalunya \\ acluai@uoc.edu
}

Recibido: 25 enero 2021; Devuelto para correcciones: 23 marzo 2021; Aceptado: 20 mayo 2021

Las alcaldesas de Robadors. Resistencia, compromiso y voz de las trabajadoras sexuales del Raval de Barcelona (Resumen)

A partir de un estudio de caso en la Calle Robadors del barrio del Raval de Barcelona, este artículo centra su atención en el lugar de las trabajadoras sexuales en el espacio urbano y en la esfera pública de la ciudad. El estudio plantea el reto que actualmente supone la supervivencia del relato de "otra" cara de Barcelona, en particular cuando el relato tiene voz de mujer que también es "otra". Enmarcado en una investigación más amplia sobre las estrategias de resiliencia y resistencia en los espacios extremos de la ciudad, el estudio de caso de la Calle Robadors incorpora la perspectiva de género en el replanteamiento de conceptos habituales en los estudios urbanos críticos, como "revanchismo" y "resistencia". El artículo aporta también la descripción del uso del documental como técnica narrativa en pro de una metodología no estigmatizante y basada en la acción comunicativa.

Palabras clave: trabajo sexual callejero; resistencia; esfera pública; Barcelona; acción comunicativa; documental.

The mayors of Robadors. Resistance, commitment and voice of sex workers from the Raval of Barcelona (Abstract)

Based on a case study on Calle Robadors in the Raval neighborhood of Barcelona, this article focuses on the place of sex workers in urban space and the city's public sphere. The study addresses the challenge currently posed by the survival of the narratives on the "other side" of Barcelona, in particular when the story has the voice of women from this other side. Framed in a broader investigation on the strategies of resilience and resistance in the extreme spaces of the city, the case study of Calle Robadors incorporates the gender perspective in the rethinking of common concepts in critical urban studies, such as "revanchism" and "resistance". The article also provides the description of the use of documentary as a narrative technique in favor of a non-stigmatizing methodology based on communicative action.

Key words: street sex work; resistance; public space; Barcelona; communicative action; documentary. 
"Las alcaldesas de Robadors" es una expresión que utilizó Janet, trabajadora sexual del Raval de Barcelona, para referirse a sus compañeras y a ella misma, en una de las conversaciones recogidas en el documental Besos en Robadors (Mata y Clua 2020), realizado en el marco de la investigación de la que se dará cuenta en presente artículo. La expresión contiene un significado que resume muchos sentimientos, todos ellos poderosos por ser positivos. El primero es la inteligencia conectada al buen humor. El segundo, la sororidad feminista. El tercero, el empoderamiento. Es fácil acercarse al tema del trabajo sexual con todos los prejuicios que los lugares comunes construyen a nuestro alrededor. Pero ¿qué ocurre cuando entras en una calle estrecha y oscura, rota por un extremo y donde escuchas historias fuera de lo común? La Calle Robadors, paradigma de la inseguridad ciudadana en tantos sentidos, te descubre que existe una lucha narrada en primera persona del plural, de la que las trabajadoras sexuales forman parte. Cuando ellas hablan sobre su vida en esta calle no lo hacen como víctimas, ni como delincuentes, ni como malas mujeres, ni como traidoras de género. Lo hacen como alcaldesas de una ciudad deseada.

La resistencia de las trabajadoras sexuales a ser expulsadas del espacio público y su lucha por continuar formando parte del barrio, ha adquirido especial significado en un contexto de "cerco" a todo aquello que transgrede la norma o cuestiona un modelo impuesto de ciudad (Masala 2015; Fernández 2011, 2014). La expulsión de las trabajadoras sexuales del Raval no puede atribuirse solamente a las lógicas del mercado que dirigen la transformación física del barrio, sino que se debe también a otros dos factores muy importantes (Manchola 2015): la existencia de una legislación y unas normativas criminalizantes y punitivas, por un lado, y, por otro lado, la prevalencia en la esfera pública de un discurso que problematiza la prostitución.

Abordar el trabajo sexual como problema tiene que ver con el hecho de que quienes lo ejercen sean mujeres. Tiene que ver también con que la expresión de la sexualidad en el espacio público se materialice (o se haga corpórea) a través de ellas (Motterle 2019). El análisis sobre el caso de Robadors que aquí se presenta parte del reconocimiento de las consecuencias que ha tenido históricamente para las mujeres el hecho de que la suma entre normatividad y moralidad se cebe en ellas (Gilligan 1985), especialmente cuando estas mujeres son trabajadoras sexuales (Federici 2006; Juliano 2001).

En las páginas que siguen se ha intentado desarrollar una propuesta metodológica y una reflexión epistemológica que contribuyan a consolidar la investigación crítica alrededor del trabajo sexual callejero en la ciudad. El artículo propone la incorporación de la perspectiva de los estudios sobre la acción comunicativa al análisis de las estrategias de resistencia de las trabajadoras sexuales en un contexto de ciudad revanchista. A las bases teóricas de la geografía urbana crítica se sumarán, así, las aportaciones de los estudios críticos en comunicación.

El artículo se estructura en cinco partes. En la primera se presentará el marco teórico de la investigación, donde se identificarán los estudios previos que se toman como referencia. En este apartado, además, se planteará una discusión alrededor de los conceptos trabajados en la investigación. Así, se propondrá una revisión de los conceptos de 
"resiliencia" y de "resistencia" en relación a las estrategias desarrolladas por las trabajadoras sexuales para mantener su trabajo en la calle. Se argumentará también la idoneidad de la incorporación del concepto de acción comunicativa al estudio de las estrategias de acceso a la esfera pública en contextos de conflicto urbano. Finalmente, el marco teórico se ocupará de la forma en que el concepto de "ciudad revanchista" ha servido de base para algunos de los trabajos sobre trabajo sexual callejero realizados desde la geografía urbana. En contraposición, se planteará un giro hacia el concepto de "ciudad de los cuidados".

Antes de abordar los aspectos metodológicos de la investigación, se dedicará un apartado a contextualizar el caso de la calle Robadors. Se explicarán, así, el fenómeno del "cerco" a Robadors y la lucha organizada que han protagonizado las trabajadoras sexuales durante la última década. En la tercera parte del artículo se explicará la metodología utilizada durante las distintas fases de la investigación. Se planteará también una reflexión, más de corte epistemológico, sobre la concepción no-extractivista y lenta del desarrollo del trabajo de campo, así como sobre la voluntad de enraizarlo en prácticas existentes del tejido social. Este planteamiento permitirá plantear la idoneidad de proponer la acción comunicativa como método que permite visibilizar discursos no hegemónicos, así como desarrollar prácticas académicas basadas en los cuidados. Tras la presentación de la metodología el artículo desarrollará una descripción más extensa del proceso de elaboración y de los primeros resultados del documental Besos en Robadors.

A modo de conclusión se propondrá una reflexión sobre el lugar del trabajo sexual callejero en la ciudad y sus repercusiones en la definición del derecho a la ciudad. Se identificarán también los retos que plantea su estudio en un momento en el que, a pesar de la deriva neoliberal, la salud del tejido social y el papel de las mujeres en los espacios informales de la ciudad aparecen con fuerza como elementos esenciales no solamente de la resistencia, sino de mera supervivencia.

\section{Marco teórico}

Sirvent y Carreras (2012) analizaron la forma en que la prostitución callejera, en aplicación de la Ordenanza Cívica de Barcelona, fue erradicada de la Ronda de Sant Antoni durante la primera década de este siglo. El repaso que hacen los autores de la persecución histórica de la prostitución en Barcelona ha servido de base para el estudio de caso que aquí se presenta, centrado en la calle Robadors. Los trabajos realizados desde el ámbito del derecho en relación a la aplicación de leyes y normativas en distintos períodos (Bodelón \& Arce 2018; Gracia 2008; López \& Mestre 2006; Mestre 2004; Nicolás 2007) constituyen también obras de referencia obligada en este artículo, puesto que ofrecen una base argumentativa sólida sobre la conexión entre los términos en que se establece el marco normativo y la articulación del ejercicio del poder entorno a los grupos de presión de cada momento.

Arantza Manchola desarrolló en su trabajo de final de grado (Manchola 2015) un análisis sobre la criminalización de la prostitución callejera a partir de la convergencia de tres factores: el tipo de legislación y de normativas que hoy día se aplican para la 
eradicación del trabajo sexual en el espacio público, las políticas urbanas que dan pie a procesos de gentrificación y la imagen pública negativa que los medios de comunicación construyen alrededor de las trabajadoras sexuales. Su trabajo (centrado en el caso del barrio de Sa Gerreria en Palma de Mallorca) planteaba la necesidad de comprender el cerco a la prostitución callejera como una suma de violencias, destacando el papel clave que tienen en ese cerco los discursos de los medios y su reproducción de estereotipos, así como su contribución al mantenimiento del estigma.

El concepto de las múltiples violencias ejercidas sobre las trabajadoras sexuales de Robadors centra la investigación que realizó la antropóloga Livia Motterle en su tesis doctoral (Motterle 2019). Su trabajo aporta una importante reflexión sobre la centralidad de la vida de las personas en esta calle del barrio del Raval, entendiendo la vida no como individualidad, sino como parte corpórea de un tejido vecinal. El trabajo de Motterle ayuda a analizar y comprender el trabajo sexual callejero desde la propia calle, y también desde "dentro", puesto que no se limita a desarrollar una etnografía al uso, pendiente de los transitares de las personas, sino que defiende la necesidad de atender sus sentires, sus sentimientos, sus lazos afectivos y su complicidad.

El estudio que Rafael Cuesta realizó sobre el barrio del Raval de Barcelona y el barrio de El Cabanyal de Valencia (Cuesta 2011) aporta otra base importante para la argumentación del presente artículo. Concretamente, interesa la forma en que el autor describe los cambios comunes que estos dos barrios han experimentado a raíz de sus respectivos procesos de gentrificación. Cuesta identificó los factores que caracterizan el barrio que desaparece en contraste con los factores que caracterizan el barrio gentrificado. Del barrio que desaparece destacan la vida en la calle, los sentimientos de arraigo, las biografías de raíces, las lógicas vecinales solidarias y las relaciones personalizadas (en contraposición con las relaciones impersonales).

Utilizando como referencia este tipo de trabajos no se pretende negar la existencia del conflicto en la realidad de Robadors. Queda lejos de la voluntad de este artículo ofrecer una visión idealizada o simplificada de la vida y de las relaciones que se tejen alrededor del trabajo sexual en esta o en cualquier otra calle. El objetivo del estudio de caso que aquí se presenta es, básicamente, dar cuenta de la existencia de otras historias y otras formas de narrar, en las que las trabajadoras sexuales tienen voz protagonista. Se trata de historias que se refieren a una forma de vivir y de entender el barrio más propias del Raval que desaparece pero que, a pesar de ello, se resisten a abandonar la ciudad.

\section{Resiliencia vs. resistencia}

El concepto de resiliencia ha sido ampliamente ultilizado desde la geografía urbana durante la última década. En el contexto anglosajón, además, este término se ha utilizado desde los gobiernos municipales y los organismos a cargo de la planificación urbana como una forma de plantear la necesidad de adaptación a los retos del futuro de la ciudad, sin cuestionar el modelo de crecimiento económico prevalente. Hace tiempo que este concepto viene siendo cuestionado por la falta de rigor con la que se ha extendido su uso (originariamente vinculado a la descripción de procesos de adaptación a situaciones extremas de cambio en 
el medio ambiente) para aplicarlo a las políticas urbanas (MacKinnon y Derickson 2013). Recientemente, además, se ha originado un interesante debate entorno a la forma en que el concepto de resiliencia niega la capacidad de agencia transformadora de las comunidades que son excluidas por el sistema (Grove et al 2020, Ranganathan y Bratman 2021).

Las trabajadoras sexuales han permanecido en el Raval durante siglos mediante el desarrollo de distintas estrategias (como, por ejemplo, la invisibilización o la clandestinidad) que les permitían sortear el señalamiento, la persecución, la sanción, el abuso o la expulsión (Sirvent y Carreras 2012). Estas formas de resiliencia, no obstante, se han erigido también como formas de fijar el estigma y de perpetuar la violencia que se ha ejercido históricamente sobre ellas. Precisamente, su situación de clandestinidad permitió y sigue permitiendo su mayor vulnerabilidad.

El estudio que aquí se presenta parte de la percepción de un cambio de estrategia protagonizado por las trabajadoras sexuales en los últimos años. Este cambio se opera a partir del momento, hace apenas una década, en que las trabajadoras sexuales abordan a cara descubierta el problema del estigma (Neira 2012; Clua 2015). Su irrupción en la esfera pública desde la lucha por los derechos laborales no las ha aislado como colectivo sectorializado, sino que ha subrayado su idetificación con la calle, con el barrio del Raval y con la ciudad de Barcelona. Este sentimiento de pertinencia es compartido por la generación de trabajadoras que llegaron a la ciudad provenientes de otras zonas de España, así como por las posteriores generaciones de trabajadoras provenientes de otros países, las cuales afrontan una estigmatización interseccional que incluye elementos como el color de piel o la falta de papeles. Esta lucha por visibilizarse y por hacer oír la propia voz se constituye a partir de su capacidad para reivindicar una identidad colectiva que les da "derecho a la ciudad" (Alsina 2015). Ello conlleva el reconocimiento de lazos afectivos de relación vecinal, así como la articulación de complicidades con nuevos movimientos sociales urbanos organizados alrededor de redes que tienen lugar hoy día tanto en espacios offline como en espacios online. En este sentido, la investigación ha puesto énfasis en esta forma de entender la resistencia en tanto que estrategia de lucha contra la desaparición de una historia colectiva y compartida.

\section{La acción comunicativa y la visibilización de las (otras) identidades}

Este artículo parte del reconocimiento de la centralidad de la acción comunicativa en la articulación de la resistencia. Se han tomado como referencia los estudios del campo de la comunicación y las ciencias sociales que han reconocido la importancia de la acción comunicativa a la hora de entender las exclusiones sociales (Couldry, Livingstone y Markham 2007).

Este marco de referencia es heredero de un debate teórico más amplio que durante las ultimas décadas ha marcado los estudios sobre la constitución de la esfera pública. La obra de Jürgen Habermas había permitido entender la acción comunicativa como un importante elemento en la comprensión de fenómenos como la integración social y sistémica (Habermas [1981] 1987). No obstante, la visión normativa de Habermas (que explica la configuración de la esfera pública desde la racionalidad del consenso) ha sido 
cuestionada desde las perspectivas que optan por definir la esfera pública como un espacio de conflicto, donde las acciones que no forman parte del discurso hegemónico tienen también sus espacios de expresión (Mouffe 2011). Es en esta "otra" esfera pública (o esferas públicas) donde se reconoce la coexistencia de distintas voces que luchan por su supervivencia, incluyendo aquellas que habitan espacios no visibles.

Para la investigación que aquí se presenta adquieren especial relevancia los trabajos que describen la esfera pública como un espacio complejo y en conflicto, donde la constitución de lugares comunes atienden a "otros" elementos "no racionales" o "no normativos" de la articulación del tejido social, como pueden ser la expresión de la estima, el cariño, el respeto, el apoyo mútuo o la amistad. Para el estudio de caso del trabajo sexual en la calle Robadors son de referencia obligada aquellos trabajos previos que han ayudado a entender el papel de las emociones en la construcción de lazos de conexión y solidaridad (De Blasio y Selva 2019).

Los fundamentos de este debate enraizado en el campo de la comunicación han sido de gran utilidad para el estudio de la calle Robadors en "clave urbana". Esta base teórica permite contraponer la ciudad cuyo discurso se expresa a través de ordenanzas municipales y planes de regeneración urbana (la ciudad que expulsa a las trabajadoras sexuales) con "la otra" ciudad en la que las mujeres de la calle forman parte de un tejido de barrio, de una historia y de una identidad colectivas. Ello permite trabajar sobre la idea de que la violencia urbana que se ha ejercido y se ejerce sobre ellas no parte solamente de su consideración como colectivo a erradicar de la calle (Fernández 2014). La violencia se ejerce también cuando, por su condición de mujeres, son consideradas tanto una amenaza al orden público y a la moral (Holgado 2001; Juliano 2002, 2009), como una lacra del orden social patriarcal. Este planteamiento enfatizará la importancia de considerar no solamente las resistencias físicas o materiales que protagonizan las trabajadoras sexuales en los espacios urbanos sino también sus resistencias simbólicas (Scott 1985, 1992) a ser estigmatizadas.

\section{Ciudad revanchista vs. ciudad de los cuidados}

Han pasado veinticinco años desde que Neil Smith (1996) acuñara la definición de revanchismo urbano como el proceso mediante el cual el capitalimo, a través de la gentrificación urbana, impone su visión excluyente de la ciudadanía mediante el escarnio público y la expulsión física de los colectivos considerados "inadaptados". En este apartado se presentarán los estudios que han abordado el tema del trabajo sexual a partir del concepto de ciudad revanchista. Destacan los trabajos que Phil Hubbard ha realizado durante casi dos décadas (Hubbard 1999, 2004a, 2004b, 2012; Hubbard, Campbell, O'Neill, Pitcher y Scoular 2007; Hubbard, Matthews, Scoular \& Agustín 2008, Hubbard \& Sanders 2003, Hubbard y Whowell 2008). Hubbard se ha interesado por la producción de espacios de trabajo sexual junto con lo que él llama "rehacer el sexo y el género en la ciudad 'revanchista' contemporánea". Este autor coincide con Laing y Cook (2014) en resaltar la importancia del carácter global de las prácticas urbanas revanchistas contra el trabajo 
sexual, ya que en la actualidad es un fenómeno que se da por igual en diferentes ciudades del mundo:

Aunque las leyes nacionales que rodean la prostitución pueden ser muy variables, con enfoques que van desde la prohibición hasta la despenalización total, pasando por la regulación más o menos higienista, a menudo existen notables similitudes y resonancias entre las ciudades en términos de la forma en que los límites de los espacios de trabajo sexual se construyen a través de políticas de contención espacial, exclusión y vigilancia (Hubbard 2016, 314).

Marilyn Adler Papayanis (2000) abrió otra línea de trabajo interesante en relación al papel que juegan las estrategias discursivas en las intervenciones urbanísticas de la ciudad revanchista. Según la autora, la clave de estas estrategias es vincular la presencia de puntos conflictivos en el centro de la ciudad (como es el caso de la zonificación del negocio del sexo en Nueva York) con "impactos secundarios" no deseados, como el aumento de la delincuencia y la disminución del valor de las propiedades en el área (Papayanis 2000, 341). Para Papayanis, es fundamental "'desenmascarar' el discurso de los impactos secundarios revelando los factores económicos y sociales entrelazados que difuminan la línea entre percepción y hecho" (Papayanis 2000, 243). Este tipo de discursos, comunes en tantas ciudades del mundo, son los que Smith identificó con el lenguaje "moral cívica" de la ciudad revanchista y con un giro populista del urbanismo (Smith 1996). Miquel Fernández (2012) analizó este mismo fenómeno, precisamente, en la Calle Robadors.

Frente a este estado de la cuestión, la investigación que aquí se presenta opta por no analizar el discurso del miedo, ni el del odio, ni el del asco (Morcillo y Varela 2021), tan extendido y fácil de propagar, sino explorar otro tipo de discurso, basado en el reconocimiento de los afectos y los cuidados que forman parte de la dimensión humana y social de las trabajadoras sexuales (Berg 2014; Desrus y Gómez 2016; Motterle 2019).

\section{Contexto}

\section{La dimensión urbana del cerco a Robadors}

Después de la eliminación del trabajo sexual en la Ronda Sant Antoni (Sirvent y Carreras 2012), la calle Robadors y la calle Sant Ramon quedaron como últimos enclaves del trabajo sexual en el Raval. En el año 2011 estas calles fueron declaradas zonas directamente afectadas por la declaración del Área de Conservación y Rehabilitación Sant RamonRobador (Masala 2015).

El Plan de Uso del Suelo de la Ciudad tipificó este espacio del Raval como un "Área de Tratamiento Específico". En la actualidad, el trabajo sexual ha desaparecido de la calle San Ramon, pero se mantiene en la calle Robadors. Antes de la pandemia por COVID-19, esta zona (específicamente, la Calle Robadors y la Plaza de Salvador Seguí) fue precisamente el escenario de muchas de las acciones realizadas en reivindicación de los derechos fundamentales (no solo laborales) de las trabajadoras sexuales. Pero para entender el significado de este enclave como espacio de resistencia es necesario explicar el contexto político municipal en el que se desarrolla la lucha. 


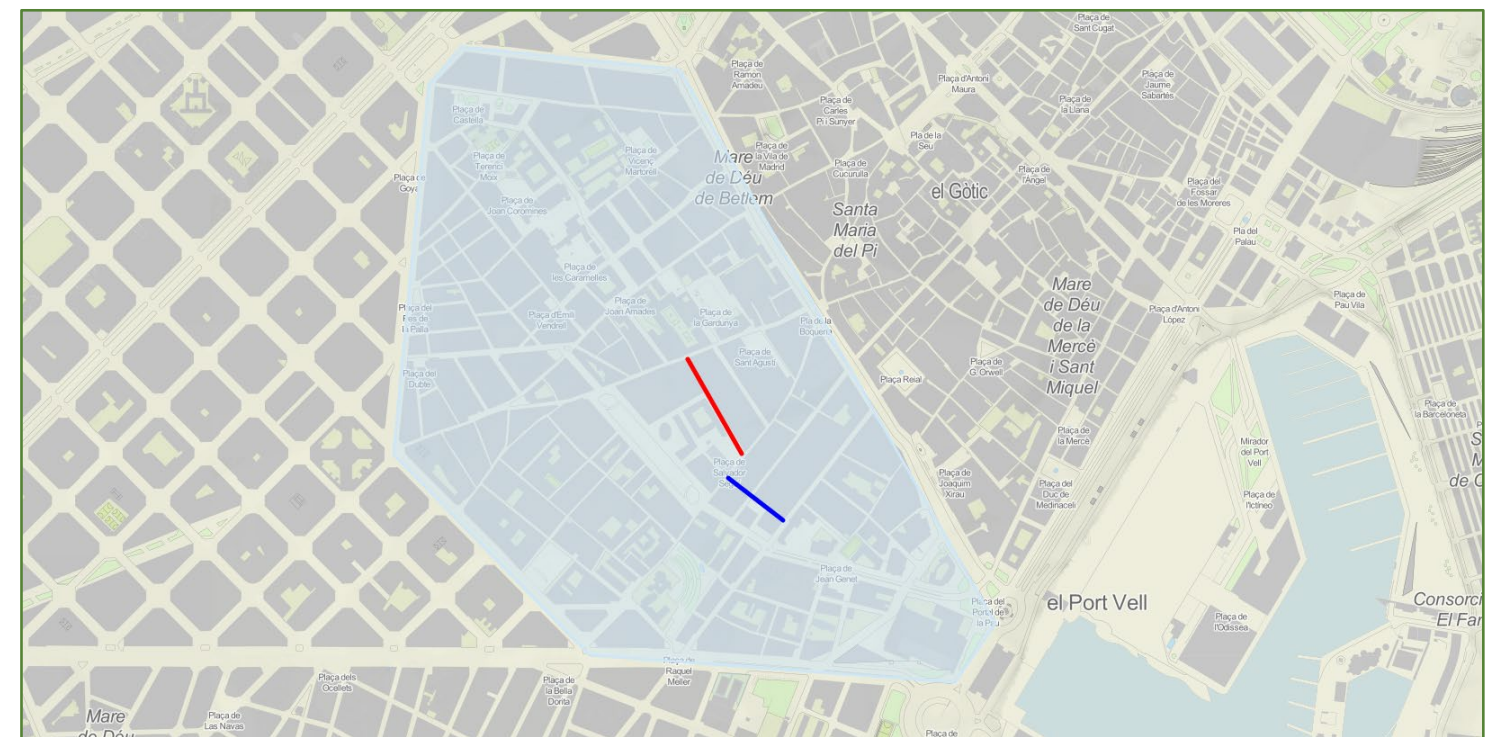

Mapa 1: Calle Robadors (en color rojo) y Calle Sant Ramon (en azul oscuro), en el Raval de Barcelona (zona sombreada)

Fuente: Elaboración propia a partir de Instamaps.

En cuanto a la segunda cuestión, si bien partíamos de las premisas sobre la transgresión de las fronteras entre sujetos y objetos en las que se basan las metodologías participativas (Ander-Egg 2003; Bergold y Thomas 2012), nos propusimos trabajar a fondo el cuestionamiento del concepto de autoridad subyacente en el hecho de "hacer partícipes" a las personas en las que se centra el estudio. De este modo, se plateó la idea de construir relatos en contextos conversacionales en base a una ruptura epistemológica con los saberes convencionales y modélicos, y desde la perspectiva feminista de la ética de los cuidados (Gilligan 2013).

Esta construcción precisaba de cinco condiciones:

1. Que en las conversaciones al menos una de las protagonistas fuera siempre una trabajadora sexual

2. Que las conversaciones tuvieran lugar en espacios comunes y significativos

3. Que la voz de las mujeres estuviera mayoritariamente representada

4. Que la relación entre las personas que protagonizaban la conversación tuviera un recorrido común, o se basara en un punto de encuentro alrededor de Robadors

5. Que la relación entre quienes estaban construyendo la conversación y quienes estuvieran registrándola tuviera lugar en un plano de igualdad

El trabajo de campo en esta tercera fase requirió el uso de herramientas de grabación distintas a las empleadas en fases anteriores. Se dejaron de lado la cámara fotográfica y la grabadora de voz para incorporar la cámara de vídeo y el micrófono direccional. Se planteó también la necesidad de llevar la grabación al terreno de sus protagonistas y no llevar a éstas al terreno de quienes estábamos tras la cámara. Esto se consiguió de dos maneras. 
Primero, atendiendo con sumo cuidado la elección del contexto donde tenían lugar las conversaciones. Éste no podía ser un espacio artificial tipo plató o laboratorio, sino que debía ser un espacio comúnmente conocido. Por ello se escogieron espacios significativos para las personas participantes y compartidos por todas ellas en sus quehaceres cotidianos $\mathrm{y}$ en sus activismos. Concretamente, las conversaciones se grabaron en cuatro espacios emblemáticos de las recientes luchas colectivas y vecinales del Raval: la Capella de la Misericordia, el Ágora Juan Andrés, El Lokal y Can 60.

Otra forma con la que se consiguió romper relaciones de poder y llevar las conversaciones al terreno de sus protagonistas fue eliminando, en la medida de lo posible, la frontera entre las participantes de las conversaciones y las personas que estaban tras la cámara. La incorporación de Pedro Mata al proyecto fue clave en este sentido. Pedro Mata es fotoperiodista y activista, miembro del colectivo Fotomovimiento, ${ }^{1}$ con fuerte compromiso con los movimientos sociales del Raval. Ha realizado la cobertura gráfica de las luchas de las trabajadoras sexuales de Robador a lo largo de los últimos diez años y es, por tanto, buen conocedor y conocido de todas las personas que conversaban. Él fue, de hecho, quien las convocó. La eliminación de la frontera se consiguió también con la participación activa en las conversaciones de quienes estábamos tras la cámara (el documental conserva, en su edición final, una de las intervenciones realizadas desde fuera del encuadre). El hecho de compartir espontáneamente (de forma no premeditada) unas bebidas o unos altramuces también facilitó la construcción de ese "meta-escenario" de conversación compartido.

Las personas que protagonizaron las conversaciones fueron nueve, tres de las cuales eran trabajadoras sexuales. Las otras seis personas tenían en común su participación activa en las protestas del barrio. En el análisis de los datos no se ha utilizado una clasificación de identidad de género binaria, con lo cual este artículo pretende evitar el clásico etiquetaje en base a la condición hombre/mujer, incorporando así la posibilidad de reconocer diversas identidades sexuales. Las nueve personas pertenecían a tres grupos de edad distintos (más de ochenta años, entre 50 y 60,y entre 30 y 40), con lo que se consiguió que al menos tres de las conversaciones fueran intergeneracionales. La decisión en torno a las combinaciones de personas para configurar los grupos conversacionales fue siempre sometida a su propia valoración. Se grabaron siete conversaciones protagonizadas por dos personas y una conversación con cuatro personas. Se elaboró un listado de temas muy básicos que guiaran las conversaciones. Estos temas apuntaban a la naturaleza emocional de los vínculos, y fueron acordados con las personas protagonistas previamente a cada una de las conversaciones. Se respetaron siempre los momentos en que éstas o quienes estaban tras la cámara se salieron del guion, priorizándose la espontaneidad de la conversación.

De todas estas conversaciones se obtuvieron 9 horas de metraje. El output final a partir de la edición del material audiovisual fue el documental Petons a Robadors. Lluites i lligams de putes i altres veïnes [Besos en Robadors. Luchas y ligazones de putas y otras vecinas] (Mata y Clua 2020), de 35 minutos de duración y centrado en las conversaciones por parejas. Tanto en la fase de edición como en la postproducción del documental se incorporaron 
especialistas que aportaron su experiencia técnica desde su "conocimiento situado" (Haraway 1988) del barrio del Raval y una total implicación con el proyecto. ${ }^{2}$

Tabla 2. Temas que guiaron las conversaciones de la fase 3

\begin{tabular}{|l|}
\hline A MODO DE GUIÓN \\
\hline ¿Cómo os conocisteis? \\
\hline ¿Quién es para ti la persona con la que conversas? \\
\hline ¿Qué es para ti el Raval? \\
\hline ¿Qué es para ti la calle Robadors? \\
\hline ¿Qué cambios has visto en el barrio? ¿Cómo ves su futuro? \\
\hline ¿Qué cambios has visto en el trabajo sexual en la calle? ¿Cómo ves su futuro?
\end{tabular}

Fuente: Elaboración propia

El planteamiento metodológico de la tercera fase de la investigación se alinea con los trabajos que han apostado por un desarrollo empírico en base a la acción comunicativa. En concreto, en este proyecto se ha abordado el uso de y acceso de la voz femenina (no necesariamente representada por mujeres) a la esfera pública digital en tanto que prácticas de empoderamiento. Para ello se tomó como referencia los trabajos entorno al acceso y uso de la tecnología desarrollados desde el feminismo por Aguado, Escofet \& Rubio (2009); Hache, Cruells y Vergés $(2006 ; 2007)$ y Navarrete (2011). Estos trabajos han permitido cuestionar los paradigmas hegemónicos sobre la brecha de género en relación a la brecha digital. Así, se ha introducido la noción de "otros espacios" y "otros usos" tecnológicos que dan cuenta de prácticas comunicativas que tradicionalmente se han visto relegadas a la esfera de lo doméstico, de la vida cotidiana o de las actividades económicas sumergidas o informales (Hache, Cruells \& Vergés 2006; 2007). Esta perspectiva permite, además, dar cuenta de la construcción de "otras narrativas" en los espacios digitales, mucho más proclives a la articulación de redes en base a la oralidad y a la solidaridad (Wajcman 2006).

\section{Otras narrativas: el documental Besos en Robadors}

El documental se elaboró durante el año 2019 a partir de la información recogida en este estudio de caso con el fin de ofrecer un testimonio sobre estas otras narrativas. El vídeo fue una combinación entre el protagonismo de las voces que se expresaban ante la cámara en primera persona y la mirada de quienes hilvanaron esta conversación coral a la hora de elaborar el guion del documental. El lenguaje audiovisual permitió explorar los matices del lenguaje no verbal entre las personas que protagonizaron las distintas conversaciones. A su vez, el lenguaje verbal, expresado en un contexto de intimidad y familiaridad, permitió

2 Ana Moriano colaboró en las tareas de montaje y edición. Carles Mestres se encargó de la postproducción. 
dejar constancia de otro tipo de temas de conversación, surgidos de las historias de vida y de los lugares comunes. Son narrativas surcadas por sentimientos, con un claro componente positivo, y que justamente por ello no acostumbran a centrar la atención de los titulares de prensa ni de las acciones comunicativas que buscan asegurarse una presencia en la esfera pública.

El lenguaje sonoro del documental permitió añadir el sonido ambiente de las conversaciones. Se consideró que ese sonido formaba parte del paisaje del Raval y por ese motivo las conversaciones se grabaron con un micrófono de cañón corto. La calidad del sonido de las conversaciones habladas se mejoró en la fase de postproducción. El documental incorpora una sola pieza musical que empieza a sonar al final, unos instantes antes de la aparición de los créditos. La canción Livin' in Carcelona (Malamara 2016) fue cedida por el grupo barcelonés de RAP combativo Malamara.

Un elemento clave en la construcción de este relato colectivo fue el refuerzo del lenguaje visual a través de las fotografías de Pedro Mata. Estas imágenes no solamente ayudan a contextualizar los relatos orales y sonoros, sino que en muchos casos aportan información y contenido, de manera que el significado de la historia queda amplificado y reforzado. Cabe subrayar que las fotografías incorporadas al vídeo formaban parte del archivo fotográfico sobre el que, a lo largo de los años, Pedro Mata había construido su portafolio alrededor de la calle Robadors.

El vídeo es, pues, un punto de encuentro de distintos relatos que hasta entonces corrían paralelos, dando vida y coherencia a las historias contadas en tanto que proyecto colectivo. Ello posibilitó la expresión de una mirada sensible que, a pesar de estar construida en base al cuidado, al reconocimiento y al respeto mutuo, parte de una firme voluntad de transformación social.

La fuerza de este contenido se hizo evidente en el momento en que el documental entró en el circuito de los actos públicos de la ciudad, a principios del 2020. En la Tabla 3 se presenta la cronología de la circulación de Besos en Robadors hasta la fecha de finalización de la redacción del presente artículo. La circulación del documental durante su casi primer año de andadura da cuenta del impacto alcanzado. ${ }^{3}$

La valoración de la realización y de la presentación del documental permite confirmar el cumplimiento de dos de los objetivos del proyecto de investigación (RESDERES) que enmarcó este estudio de caso. El primer objetivo era el de implicar a distintos agentes del barrio, así como (en el caso de Robadors) a las trabajadoras sexuales. El segundo objetivo era el de realizar la devolución del conocimiento generado por la investigación para su contribución al debate social y a la lucha contra el estigma en la ciudad de Barcelona. La mayoría de los actos públicos en los que se presentó el documental contaron con la presencia de algunas de las protagonistas del vídeo y muchos de ellos tuvieron lugar en espacios emblemáticos de los movimientos de base de la ciudad (ver Imagen 1), donde el video tuvo una excelente acogida. Cabe destacar el interés que generó

\footnotetext{
${ }^{3}$ El listado de la tabla no incluye los actos que se suspendieron debido a las restricciones decretadas durante el estado de alarma provocado por la pandemia COVID-19 a partir del mes de marzo de 2020.
} 
Besos en Robadors en otros circuitos, como la XXI edición del Festival de Cine Migrante, que incluyó el documental en su programa.

Otro resultado importante a destacar aquí, a parte del impacto e interés generado por el vídeo, es la reapropiación del documental por parte de colectivos de la ciudad. El pase del vídeo en espacios abiertos al público sirvió en algunos casos para la recaudación de fondos que se destinaron a la autogestión de estos espacios, o bien a la financiación de cajas comunes y causas colectivas. El documental se publicó, de hecho, con licencia Creative Commons a fin de permitir este uso desde su libre circulación.

Imagen 1: Carteles de algunos de los pases en Barcelona del documental Besos en Robadors

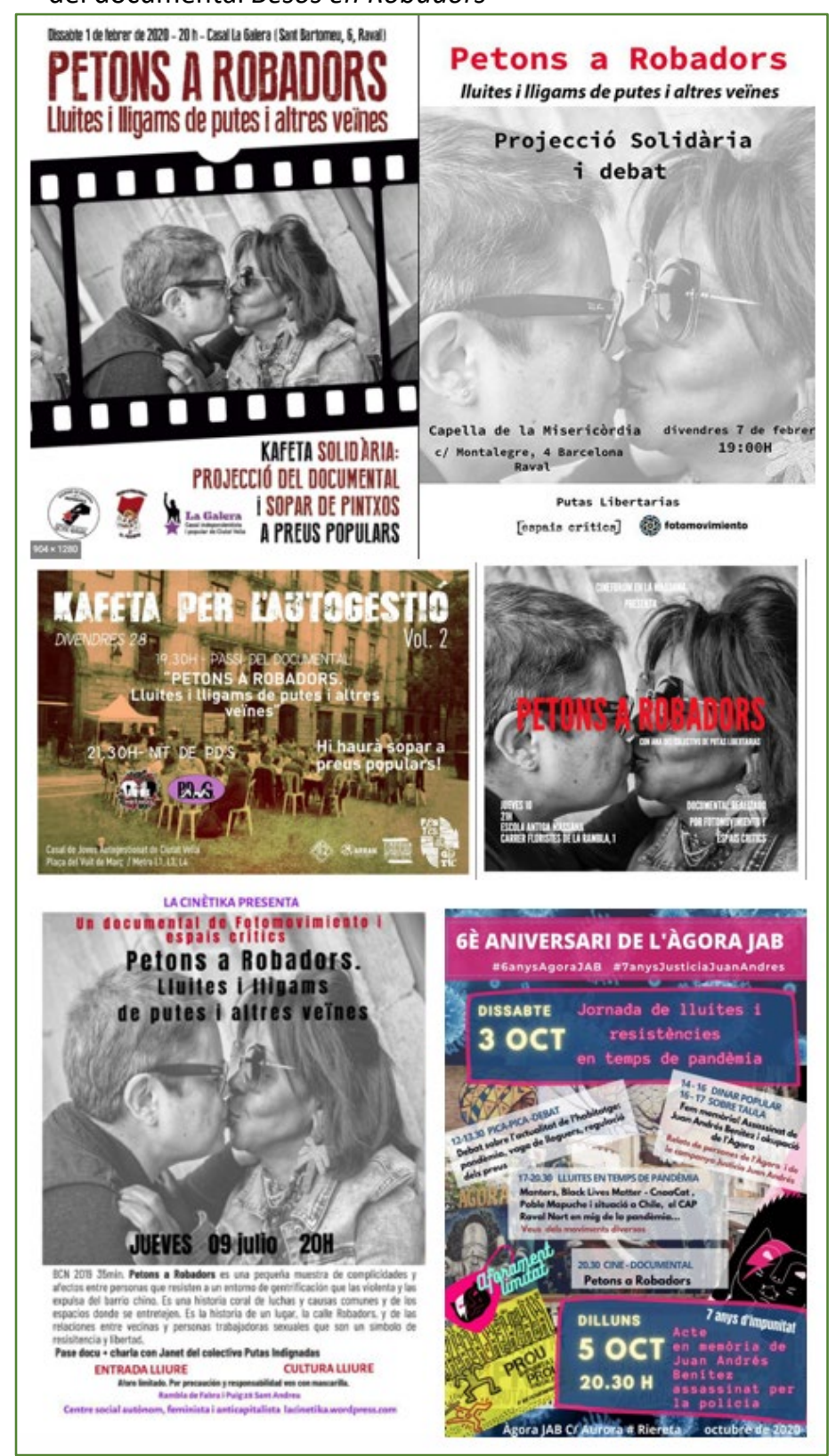

Fuente: Elaboración propia a partir de los carteles difundidos por las entidades organizadoras de los eventos. Fotografía en blanco y negro de Pedro Mata. 
Tabla 3. Cronología de la circulación por Barcelona de Besos en Robadors en 2020

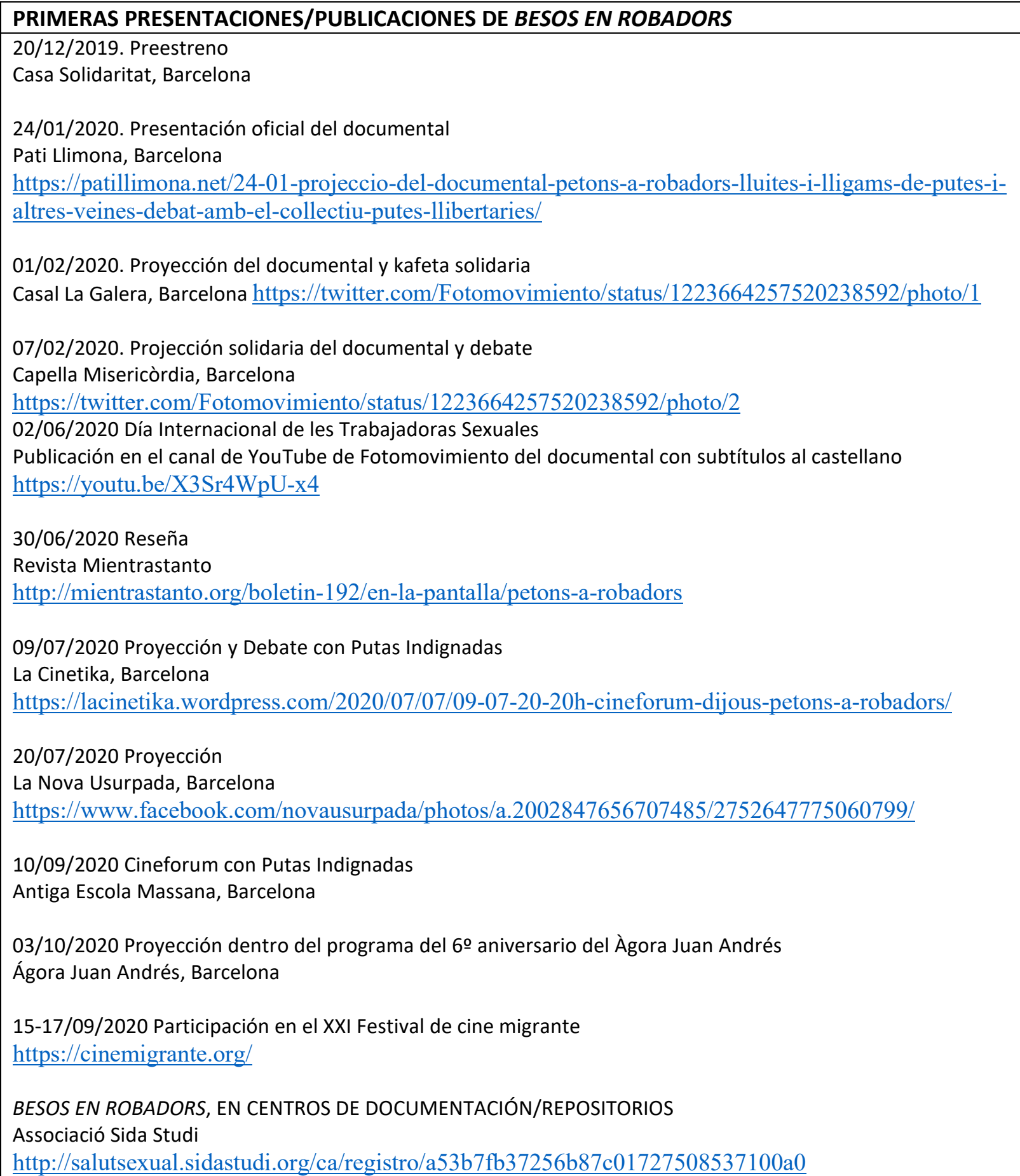

Fuente : Elaboración propia a partir de datos compartidos con Pedro Mata.

De los datos obtenidos en esta tercera fase de la investigación se evidencia otro resultado importante: la lucha por el reconocimiento de los derechos fundamentales de las trabajadoras sexuales gana fuerza en el momento en que se expresa fuera de los espacios de confrontación. De ahí surge la necesidad de reconocer la importancia de los espacios compartidos de lucha vecinal, construidos en base a unas narrativas que empiezan a priorizar la defensa del papel indispensable que juegan los cuidados en un entorno revanchista. 
Estas otras narrativas se construyen en base a la vida cotidiana, dando pie a historias donde las emociones juegan un papel político primordial. Los relatos resultantes ponen sobre la mesa una imagen de las trabajadoras sexuales que no coincide con la imagen estereotipada que se recrea en la esfera pública. Son narrativas que rompen con el estigma. La gran fuerza de estos relatos radica precisamente en la forma en que las trabajadoras sexuales ( $\sin$ actuar frente a la cámara sino precisamente mostrándose tal y como son) le dan la vuelta a la imagen que han construido de ellas los medios de comunicación. Aparece así una dimensión humana mucho más compleja, llena de matices y que trasciende la individualidad. El saberse una persona que es mucho más que una mera imagen de sí misma es un idea de la que se habla explícitamente en una de las conversaciones que se reproducen en el documental: "Parezco muy valiente, pero no es verdad" (Ana, en Mata y Clua 2020, min. 07:05). En este caso, la constatación de la propia vulnerabilidad adquiere, sin embargo un significado contrario en el vídeo, puesto que éste pone en evidencia la idea de que la fuerza de las trabajadoras sexuales reside no tanto en el reconocimiento de la capacidad combativa entendida a título personal sino en el reconocimiento del apoyo mutuo que se genera entre compañeras y dentro del tejido social del vecindario.

\section{A modo de conclusión}

El estudio del que surge el presente artículo se ha centrado en la búsqueda y análisis de narrativas contra-hegemónicas en las zonas intersticiales de la ciudad. Los procesos de transformación del barrio del Raval de Barcelona han puesto en jaque su imagen en tanto que espacio de transgresión, así como el sentido de pertenencia y de identificación de sus habitantes con una cultura de barrio obrero enraizada en la solidaridad (Cuesta 2011; Fernández 2011, 2014). En el caso de la calle Robadors, las narrativas que tienen voz de mujer se han articulado con especial fuerza (sobre todo a raíz de la aplicación de la Ordenanza Cívica en Barcelona) alrededor de la figura de la trabajadora sexual. La investigación concluye que la voz de las trabajadoras sexuales no es una voz aislada, sino articulada en el tejido social del barrio, de su memoria histórica y de sus luchas vecinales.

Miquel Fernández analizó hace unos años la forma en que el "cerco urbano" al barrio del Raval amenaza con "matar al Chino" (Fernández 2012, 2014). Al Chino (nombre popular que ha recibido el Raval históricamente) no solo se le "mata" cuando se aplican planes urbanísticos o normativas municipales y leyes estatales donde la prostitución no tiene cabida, sino también cuando su denominación popular pierde significado por dejar de ser un referente. En este punto, la voz de las trabajadoras sexuales que ejercen en la calle no solo es importante a la hora de reconocer su derecho a ejercer su profesión, sino también a la hora de reconocer su identidad como vecinas del barrio, desde donde pueden reclamar su pertenencia (y su derecho) a la ciudad (Alsina 2015). Reconocerlas solamente como víctimas de un entramado capitalista-patriarcal las despoja de su entidad y capacidad de agencia política. Hacer que todo debate gire alrededor del grave problema de la prostitución derivada de la trata de seres humanos con fines de explotación sexual resulta un peligroso ejercicio de subyugación de las mujeres (sobre todo las más vulnerables) a la anomía. Es interesante, por tanto, subrayar la efectiva simplicidad con la que los discursos a 
favor de la expulsión de las prostitutas del barrio optan por la estrategia de relacionar prostitución y trata. Si ubicamos este fenómeno en un contexto histórico más amplio podemos visualizar claramente la manifestación política de un revanchismo feminizado. Los discursos a favor de la expulsión de las trabajadoras sexuales provienen no solo de las normas que regulan el ejercicio de la prostitución en la ciudad sino del tipo de moralidad de largo recorrido que subyace en los discursos hegemónicos.

El frágil equilibrio del ecosistema del Chino, donde el trabajo sexual había encontrado históricamente su nicho, se está quebrando a velocidad creciente. Las trabajadoras sexuales de la calle (todas sin excepción, sin importar si son víctimas de trata o no) actualmente aparecen como el residuo del desorden, lo que las hace más vulnerables a ser "barridas" del mapa o a quedar en situación de desamparo legal. En este proceso no se tiene suficientemente en cuenta el hecho de que no solamente se expulsa del barrio a las mujeres que "hacen la calle" en abstracto, sino a sus transitares, a sus historias personales, a sus rasgos generacionales, a sus memorias del lugar, a sus condiciones de inmigradas, de mujeres racializadas, a sus sororidades, así como a toda posibilidad de arraigo y apego y, en consecuencia, de participación en la construcción de la ciudad.

El trabajo que se ha presentado en este artículo pretende ser una muestra de la posibilidad de desarrollar una investigación sobre colectivos vulnerables de mujeres que se aleje de las prácticas hegemónicas estigmatizantes o abusivas, así como de la difusión de saberes normativos, también desde la academia. Desde su apuesta por el uso de una metodología no extractivista, el proyecto ha intentado ofrecer un marco de exploración para el uso de la acción comunicativa como herramienta de análisis y, a la vez, de empoderamiento.

La investigación iniciada con este estudio de caso de la Calle Robadors permite dar cuenta de las posibilidades que se abren ante el uso del lenguaje audiovisual en proyectos de investigación comprometidos con la transformación social. En este sentido, queda pendiente explorar y profundizar en temas como el de la construcción de la mirada, el trabajo colaborativo desde las primeras fases de diseño del proyecto, la socialización de saberes alrededor de la comunicación audiovisual, incluyendo el aprendizaje de la elaboración de guiones y de la edición de videos, así como la preparación y adecuación de contenidos para su difusión en una esfera pública donde las redes sociales (bien con sus raíces físicas en la ciudad, bien con sus ramificaciones virtuales) abren nuevos espacios para la comunicación y la conectividad. Trabajos como los de Artigas (2021) pueden ayudar a completar la construcción de un marco teórico que contemple estos aspectos aplicados a la acción comunicativa con voluntad emancipadora.

El artículo plantea también la necesidad de abrir debates entorno a la ruptura epistemológica que la perspectiva de género plantea a los estudios urbanos. La incorporación de la ética de los cuidados al trabajo académico con colectivos vulnerables ofrece la posibilidad de afrontar los nuevos retos que las crisis planetarias (como la desatada por la pandemia del COVID-19) plantearán sin duda a la evolución de las ciudades y de los derechos fundamentales de las ciudadanas y los ciudadanos. 


\section{Bibliografía}

Aguado, Gemma, Escofet Anna,y Rubio María José. 2009. "Empoderamiento, tecnologías de la información y la comunicación y género. Una aproximación conceptual.” En Identidades femeninas en un mundo plural, editado por María Elena Jaime de Pablos. Barcelona: Arcibel Editores, pp. 11-18

Alsina, Carla. 2015. "Technopolitics, sexual rights and sex workers' right to the city", en Global Information Society Watch 2015. Sexual Rights and the Internet, editado por A. Finlay. APC \& HIVOS. https://www.giswatch.org/2015-sexual-rights-and-internet [Consultado el 11 de Diciembre de 2019]

Ander-Egg, Ezequiel. 2003. Repensando la investigación-acción-participativa. Buenos Aires: Lumen

Artigas, Xavier. 2021. Documental social: De la Transformación a la Emancipación. Universitat Oberta de Catalunya (forthcoming).

Berg, Heather. 2014. Working for love, loving for work: Discourses of labor in feminist sexwork activism. Feminist Studies, 40(3), 693-721.

Bergold, Jarg y Thomas, Stefan. 2012. "Participatory Research Methods: A Methodological Approach in Motion". Historical Social Research / Historische Sozialforschung, 37(4 (142), 191-222.

Bodelón, Encarna y Arce, Paula. 2018. “La reglamentación de la prostitución en los ayuntamientos: una técnica de ficticia seguridad ciudadana". Crítica Penal y Poder, 15, 71-89, OSPDH. Universidad de Barcelona.

Couldry, Nick, Livingstone, Sonia y Markham, Tim. 2007. "Connection or Disconnection?: Tracking the Mediated Public Sphere in Everyday Life.", en Media and Public Spheres, editado por Richard Butsch 28-42. Basingstoke, UK/ New York, USA: Palgrave Macmillan.

Clua, Anna. 2018. "Muchas gracias por su colaboración" Qué piensan las trabajadoras sexuales de su participación en investigaciones académicas". Simposio Internacional Prostituciones Mediatizadas. Universidad Complutense de Madrid: 21-22 de Mayo de 2018

Clua, Anna. 2015. "La batalla simbólica de las prostitutas: El papel de la comunicación". Revista Internacional de Comunicación y Desarrollo, 1, 139-150.

Clua, Anna y Moré, Joaquim. 2017. "El debate público sobre prostitución: Estudio de caso de la repercusión de un programa televisivo en la esfera Twitter". En Ciberpolítica. Gobierno abierto, redes, deliberación, democracia, editado por Ramón Cotarelo. \& Javier Gil, 291-308. Madrid: Instituto Nacional de Administración Pública.

Cuesta, Rafael. 2011. "Procesos de asimilación y resistencia urbanas en el marco de la globalización. Aproximaciones al barrio Chino de Barcelona y al barrio del Cabanyal de Valencia". Revista Nuevas Tendencias en Antropología, (2), 104-146.

De Blasio, Emiliana y Selva, Donatella. 2019. "Emotions in the Public Sphere: Networked Solidarity, Technology and Social Ties", en Emotions and Loneliness in a Networked Society, editado por Bianca Fox, 13-44. Wolverhampton, UK: Palgrave Macmillan 
Desrus, Bénédicte y Gomez, Celia. 2016. Las amorosas más bravas. México: Los Libros del Sargento.

Federici, Silvia. 2006. "Prostitution and Globalization: Notes on a Feminist Debate". En Poverty and the Production of World Politic, editado por Matt Davies \& Magnus Ryner , 113-136. Palgrave Macmillan, London.

Fernández, Miquel. 2011. "El asedio al Raval: Una aproximación al pasado y presente de las transformaciones urbanísticas del barrio barcelonés". Oñati Socio-Legal Series, 1 (2), $1-25$.

Fernández, Miquel. 2012. "Usos de l'estigma. El paper de la prostitució en la revalorització urbanística de l'Illa Robador a la ciutat de Barcelona”. Quaderns-e de l'Institut Català d'Antropologia, 17 (2), 86-98.

Fernández, Miquel. 2014. Matar al Chino: Entre la revolución urbanística y el asedio urbano en el barrio del Raval de Barcelona. Barcelona: Virus.

Garcia Grenzner, Joana. Nd. “2015-2016: efervescencia feminista social y política para frenar la restauración del 78". Anuario de los Movimientos Sociales 2015-2016. Fundación Betiko. http://fundacionbetiko.org/wpcontent/uploads/2017/02/03_garcia_feminismo.pdf [Consultado el 23 de abril de 2020]

Gilligan, Carol. 1985. La moral y la teoría: Psicología del desarrollo femenino. México D.F: Fondo cultural económico de México

Gilligan, Carol. 2013. Joining the resistance. John Wiley \& Sons.

Gracia, Ricard. 2008. "Convivencia ciudadana, prostitución y potestad sancionadora municipal: El caso de Barcelona". QDL, 17, 99-133.

Grove, Kevin; Cox, Savannah y Barnett, Allain. 2020. Racializing resilience: assemblage, critique, and contested futures in greater Miami resilience planning. Annals of the American Association of Geographers, 110(5), 1613-1630.

Habermas, Jürgen. [1981] 1987. Teoría de la acción comunicativa, I. Racionalidad de la acción y racionalización social. Madrid: Taurus.

Hache, Alexandra, Cruells, Eva y Vergés, Núria. 2006. “CodiLela: Recerca sobre l’accés, ús i pràctiques de les tecnologies per part de les dones a Catalunya." DonesTech. https://www.donestech.net/files/codilela.pdf [Consultado el 10 de Diciembre de 2020]

Hache, Alexandra, Cruells, Eva \& Vergés, Núria. . 2007. “Código Lela: Investigación sobre el acceso, usos y prácticas de las tecnologías por parte de las mujeres en el estado español." DonesTech. https://www.donestech.net/files/codigolela.pdf [Consultado el 10 de Diciembre de 2020]

Haraway, Donna. 1988. Situated knowledges: The science question in feminism and the privilege of partial perspective. Feminist studies, 14(3), 575-599

Holgado, Isabel. 2001. "Las nuevas retóricas de la inmigración femenina: la prostitución en las calles de Barcelona". Scripta Nova: Revista Electrónica de Geografía y Ciencias Sociales, 94 (100) http://www.ub.es/geocrit/sn-94-100.htm [Consultado el 12 de Junio de 2019] 
Hubbard, Phil. 1999. Sex and the city: Geographies of prostitution in the urban West. Aldershot: Ashgate.

Hubbard, Phil. 2004a. "Revenge and injustice in the revanchist city: Uncovering masculinist agendas". Antipode, 36 (4), 665-686.

Hubbard, Phil. 2004b. "Cleansing the metropolis: Sex work and the politics of zero tolerance". Urban Studies, 41 (9), 1687-1702.

Hubbard, Phil. 2012. Cities and sexualities. London: Routledge.

Hubbard, Phil. 2016. "Sex work, urban governance and the gendering of cities", en The Routledge Research Companion to Geographies of Sex and Sexualities, editado por Gavin Brown \& Kath Browne, 313-320. London: Routledge.

Hubbard, Phil; Campbell, Rosie, O'Neill, Maggie, Pitcher, Jane y Scoular, Jane 2007. "Prostitution, gentrification and the limits of neighbourhood space", en Securing an Urban Renaissance, editado por Rowland Atkinson, \& Gesa Helms, 203-217. Bristol: Policy Press.

Hubbard, Phil, Matthews, Roger, Scoular, Jane y Agustín, Laura. 2008. “Away from prying eyes? The urban geographies of adult entertainment”. Progress in Human Geography, 32 (3), 363-381.

Hubbard, Phil y Sanders, Teela 2003. "Making space for sex work: female street prostitution and the production of urban space". International Journal of Urban and Regional Research, 27 (1), 75-89.

Hubbard, Phil y Whowell, Mary. 2008. "Revisiting the red light district: Still neglected, immoral and marginal?". Geoforum, 39 (5), 1743-1755.

Juliano, Dolores. 2009. “Delito y pecado. La transgresión en femenino”. Política y sociedad, 46(1), 79-95.

Juliano, Dolores. 2002. La prostitución: el espejo oscuro. Barcelona: Icaria

Juliano, Dolores. 2001. “Modelos de género a partir de sus límites: la prostitución”, en Multiculturalismo y género. Un estudio interdisciplinar, editado por Nash, Mary \& Diana Marre, 87-115. Barcelona: Bellaterra

Laing, Mary y Cook, Ian. 2014. "Governing sex work in the city". Geography Compass, 8, 505515.

López, Magdalena y Mestre, Ruth. 2006. Trabajo sexual: Reconocer derechos. Valencia: Ediciones La Burbuja.

MacKinnon, Danny y Derickson, Kate Driscoll (2013). From resilience to resourcefulness: A critique of resilience policy and activism. Progress in human geography, 37(2), 253270.

Malamara. 2016. “Livin' in Carcelona”, en La Palabra EP \#4F/2016, released February 4, 2016. https://malamara.bandcamp.com/track/livin-in-carcelona

Manchola, Arantza. 2015. Urbanismo especulativo, medios de comunicación y administración pública: juntos contra la prostitución. [Trabajo Final de Grado. Grado en Trabajo Social] Universitat de les Illes Balears. https://dspace.uib.es/xmlui/bitstream/handle/11201/1529/Manchola_Di\%cc\%81az_Arantz a TFG.pdf?sequence $=1 \&$ isAllowed $=y$ 
Masala. 2015. "Cerco a Robador". Masala Revista d'informació, denúncia i crítica social a Ciutat Vella, 71 (Desembre 2015). https://masala.cat/cerco-a-robador/ [Consultado el 15 de octubre de 2020]

Mata, Pedro y Clua, Anna (2020) Petons a Robadors. Lluites i lligams de putes i altres veïnes. Fotomovimiento y Espais Crítics. https://youtu.be/X3Sr4WpU-x4

Mestre, Ruth. 2004. "Las caras de la prostitución en el estado español: Entre la ley de extranjería y el código penal", en Trabajadoras del sexo: Derechos, migraciones y tráfico en el siglo XXI, editado por Raquel Osborne, 245-261. Barcelona: Bellaterra.

Miralles, Ana María. 2001. Periodismo, opinión pública y agenda ciudadana. Bogotá: Editorial Norma

Morcillo, Santiago y Varela, Cecilia. 2021. ¡ Puaj! Las retóricas del asco en el movimiento abolicionista de la prostitución en Argentina. Revista Estudos Feministas, 29(1).

Motterle, Livia. 2019. Y tenía corazón. Trabajo sexual, violencias y resistencias entre carne y piedra en el Raval de Barcelona. [Tesis Doctoral] Universitat de Barcelona. http://diposit.ub.edu/dspace/bitstream/2445/137283/5/LIVIA\%20MOTTERLE_TESIS.pd $\underline{f}$

Mouffe, Chantal. 2011. On the political. Routledge.

Navarrete, Ana. 2011. "Diferentes, desiguales y desconectadas. ¿Quién es quién en las industrias tecnológicas?." Repositorio RuiDeRa. https://ruidera.uclm.es/xmlui/bitstream/handle/10578/3745/fi 1398191210diferentes $\% 20$ desiananavarrete.pdf? sequence $=1 \&$ isAllowed $=y$ [consultado el 10 de Diciembre de 2020]

Neira, Montse. 2012. Una mala mujer. La prostitución al descubierto. Barcelona: Plataforma Editorial.

Nicolás, Gemma. 2007. La Reglamentación de la prostitución en el Estado Español: Genealogía jurídico-feminista de los discursos sobre prostitución y sexualidad. Tesis Doctoral. Barcelona: Universitat de Barcelona, Facultat de Dret, Departament de Dret Penal i Ciències Penals.

Papayanis, Marilyn. 2000. "Sex and the Revanchist City: Zoning out Pornography in New York". Environment and Planning D. Society and Space, 18 (3), 341-353.

Ranganathan, Malini y Bratman, Eve (2021). From urban resilience to abolitionist climate justice in Washington, DC. Antipode, 53(1), 115-137.

Scott, James. 1985. Weapons of the Weak: Everyday Forms of Peasant Resistance. London: Yale University Press.

Scott, James. 1992. Domination and the Arts of Resistance: Hidden Transcripts. London: Yale University Press.

Sirvent, Eva y Carreras, Jordi. 2012. Dones del carrer: Canvi urbanístic i treball sexual a Barcelona (2005-2009). Barcelona: Generalitat de Catalunya, Departament de Cultura.

Smith, Neil. 1996. The New Urban Frontier: Gentrification and the Revanchist City. London: Routledge.

Wajcman, Judy. 2006. El tecnofeminismo. Madrid: Cátedra 
(C) Copyright Anna Clua, 2021

(C) Copyright: Scripta Nova, 2021.

Ficha bibliográfica:

CLUA, Anna. Las alcaldesas de Robadors. Resistencia, compromiso y voz de las trabajadoras sexuales del Raval de Barcelona. Scripta Nova. Revista Electrónica de Geografía y Ciencias Sociales. Barcelona: Universitat de Barcelona, vol. 25, Núm. 2 (2021), p. 37-56[ISSN: 1138-9788]

DOI: $10.1344 / \operatorname{sn} 2021.25 .33353$ 